\title{
Estrogens maintain skeletal muscle and satellite cell functions
}

\section{Yuriko Kitajima and Yusuke Ono}

Department of Stem Cell Biology, Atomic Bomb Disease Institute, Nagasaki University Graduate School of Biomedical Sciences, Nagasaki, Japan
Correspondence should be addressed to $\mathrm{Y}$ Ono

Email

yusuke-ono@nagasaki-u.ac.jp

\begin{abstract}
Estrogens have crucial roles in an extensive range of physiological functions regulating cellular proliferation and differentiation, development, homeostasis, and metabolism. Therefore, prolonged estrogen insufficiency influences various types of tissues expressing estrogen receptors (ERs). Although ERs are expressed in skeletal muscle and its stem cells, called satellite cells, how prolonged estrogen insufficiency affects their function remains unclear. In this study, we investigated the effect of estrogen reduction on muscle in young ovariectomized (OVX) female mice. We found that reduced estrogens resulted in muscle atrophy in a time-dependent manner. Muscle force generation was reduced in OVX mice. Interestingly, prolonged estrogen insufficiency shifted fiber types toward faster myosin heavy chain isoforms. The number of satellite cells per isolated myofiber was unchanged, while satellite cell expansion, differentiation, and self-renewal were all markedly impaired in OVX mice. Indeed, muscle regeneration was significantly compromised in OVX mice. Taken together, our results demonstrate that estrogens are essential for comprehensively maintaining muscle function with its insufficiency affecting muscle strength and regeneration in young female mice.
\end{abstract}
Key Words
- estrogens
- skeletal muscle
- muscle atrophy
- satellite cells
- females

\section{Introduction}

Estrogens have crucial roles in an extensive range of physiological functions regulating cellular proliferation and differentiation, development, homeostasis, and metabolism (Mauvais-Jarvis 2011). Estrogen insufficiency is caused by a variety of factors such as menopause, surgical ovariectomy, and chemotherapy. In females, insufficient energy intake leads to body weight loss that also causes hypoestrogenic menstrual abnormalities. The female athlete triad, low energy availability, menstrual dysfunction, and low bone mineral density, is prevalent in sports activities, particularly endurance sports such as marathons as well as aesthetic sports such as gymnastics and ballet (Matzkin et al. 2015). ERs are expressed in a variety of tissues including bone and muscle (Mauvais-Jarvis 2011). Estrogens play an important role in the maintenance of mineral bone density by inhibition of bone remodeling and resorption as well as increasing bone formation. Indeed, prolonged estrogen insufficiency leads to loss of bone mineral density, resulting in fragility fractures. For example, a cross-sectional study showed that menstrual dysfunction may be a possible risk factor contributing to stress fractures in female endurance athletes (Duckham et al. 2012). Furthermore, a prospective study reported that teenage female athletes with menstrual dysfunction had a greater risk of musculoskeletal injury (Rauh et al. 2010).

Accumulating evidence suggests sex-specific differences in muscle homoeostasis. Although the effects of androgens on muscle function have been evaluated extensively, the roles of estrogens in muscle have been largely unknown. Recent studies have revealed that the levels of estrogens

Published by Bioscientifica Ltd 
are associated with muscle mass (McClung et al. 2006, Sitnick et al. 2006, Sugiura et al. 2006, Pollanen et al. 2011). Ovariectomized (OVX) mice fail to fully recover muscle mass after hindlimb suspension-induced muscle atrophy followed by reloading (McClung et al. 2006). This failure to recover muscle mass in OVX mice may be associated with reduced p70S6K signaling (McClung et al. 2006, Sitnick et al. 2006). Moreover, treatment with estrogens attenuates immobilization-induced muscle atrophy, even in male rats (Sugiura et al. 2006). These findings indicate that estrogens have a role in alleviating disuse-induced muscle atrophy and promoting regrowth after reloading. However, the effect of prolonged estrogen insufficiency on muscle remains to be fully understood.

Muscle tissue stem cells, called satellite cells, play crucial roles in providing myonuclei for postnatal muscle growth as well as in muscle maintenance, repair, regeneration, and hypertrophy in adults (Dumont et al. 2015). Thus, understanding the regulation of satellite cells is critical to elucidate the mechanisms in skeletal muscle maintenance. Several studies have reported that estrogens appear to favor muscle regeneration after injury (Diel 2014). Estrogens attenuate exercise-induced muscle damage by downregulation of inflammatory responses (Tiidus et al. 2001), increase the number of satellite cells, and stimulate satellite cell proliferation after running exercise (Enns \& Tiidus 2008). However, whether extrinsic factors influence satellite cell activity or the intrinsic potential of satellite cells is affected by estrogen insufficiency remains unclear.

In this study, we explored the impact of prolonged estrogen reduction on muscle and satellite cells in young OVX female mice. Estrogen insufficiency resulted in muscle atrophy, reduced muscle force generation, and a shift to a faster fiber-type distribution. Although the number of satellite cells per isolated single myofiber was unaltered, population expansion, myogenic differentiation, and self-renewal of satellite cells were significantly reduced in OVX mice. Indeed, muscle regeneration was impaired by a reduction of estrogen levels. Therefore, these results indicate that estrogens are crucial to maintain the functions of both muscle and satellite cells in young female mice.

\section{Materials and methods}

\section{Antibodies and reagents}

Antibodies and reagents were obtained from the following sources: mouse anti-type IIa myosin heavy chain
(MyHC) (SC-71) and mouse anti-type IIb MyHC (BF-F3) antibodies were purchased from Deutsche Sammlung von Mikroorganismen (Braunschweig, Germany). A rat anti-laminin $\alpha 2$ antibody was obtained from Alexis (San Diego, CA, USA). A goat anti-collagen type 1 antibody was purchased from Southern Biotech (Birmingham, AL, USA). Slow-release $17 \beta$-estradiol pellets $(0.01 \mathrm{mg} / 60$ days) were purchased from Innovative Research of America (Sarasota, FL, USA). Mounting medium containing DAPI for nuclear staining and an M.O.M. kit were obtained from Vector Laboratories (Burlingame, CA, USA).

\section{Animals}

Animal experimentation was approved by the Experimental Animal Care and Use Committee of the Nagasaki University, Nagasaki, Japan. Six-week-old female C57BL/6 mice (Charles River Laboratories) were ovariectomized under anesthesia. The slow-release $17 \beta$-estradiol pellets were implanted at the same time as OVX for estrogen replacement as described previously (Kitajima et al. 2015). Sham-operated mice were used as a control. All mice were killed at 2, 4, 8, and 24 weeks after surgery.

\section{Grip test}

Maximal limb muscle force was measured by a Grip Strength Meter (Columbus Instruments, Columbus, $\mathrm{OH}$, USA). Three sets of ten successive measurements were carried out to assess forelimb grip strength. The maximum values in three sets of experiments were used for data analysis.

\section{Muscle regeneration}

To induce muscle injury, $50 \mu \mathrm{L}$ of $10 \mu \mathrm{M}$ cardiotoxin (CTX, Sigma-Aldrich) was injected intramuscularly into the tibialis anterior (TA) muscle of anesthetized mice using a $29 \mathrm{G}$ $1 / 2$ insulin syringe. Regenerating muscles were isolated at 14 days after CTX injection. Transverse sections of the muscles were prepared using a cryostat and immunostained.

\section{Satellite cell isolation and culture}

Extensor digitorum longus (EDL) muscles were isolated and then digested by type I collagenase as described previously (Collins \& Zammit 2009). Satellite cells were obtained from the isolated myofibers and cultured in a

Published by Bioscientifica Ltd 
mitogen-rich medium (GlutaMax DMEM supplemented with $10 \%$ horse serum, $0.5 \%$ chicken embryo extract (CEE, US Biologica, Salem, MA, USA), and 1\% penicillinstreptomycin) at $37^{\circ} \mathrm{C}$ with $5 \% \mathrm{CO}_{2}$.

\section{Immunostaining}

Immunocytochemical analysis of satellite cells associated with myofibers was performed as described previously (Masuda et al. 2015). Samples were incubated with primary antibodies at $4^{\circ} \mathrm{C}$ overnight following blocking/ permeabilization with phosphate-buffered saline containing $0.3 \%$ Triton X-100 and 5\% goat or swine serum for $20 \mathrm{~min}$ at room temperature.

Immunohistochemistry of muscle tissues was performed as described previously (Ono et al. 2011). Muscle tissues were isolated from the TA muscle, immediately frozen in 2-methylbutane cooled in liquid nitrogen, and stored at $-80^{\circ} \mathrm{C}$ before cryosectioning. Frozen cross-sections of the TA muscle were fixed in $4 \%$ paraformaldehyde, blocked with M.O.M. (Vector Laboratories), and incubated with primary antibodies at $4^{\circ} \mathrm{C}$ overnight.

Immunostained samples were visualized using appropriate species-specific Alexa Fluor fluorescenceconjugated secondary antibodies (Life Technologies). The samples were viewed under either an IX83 microscope (Olympus) or an all-in-one Keyence fluorescence microscope (Keyence, Osaka, Japan). Images were optimized globally and assembled into figures using Adobe Photoshop.

\section{Statistical analysis}

Significant differences were determined using the Student's $t$-test or one-way ANOVA with Fisher's least significant difference (LSD) test, where appropriate. A $P$-value of $<0.05$ was considered as statistically significant. All data are expressed as mean \pm s.E.M.

\section{Results}

\section{Ovariectomy leads to a decrease in uterine size and an increase in body weight}

To induce estrogen insufficiency in vivo, young C57BL/6 female mice were ovariectomized at 6 weeks of age and killed at 2, 4, 8, and 24 weeks after ovariectomy. Sham-operated mice were used as a control (Fig. 1A).
We found an atrophic uterus in OVX mice and a gradual increase in the body weight of OVX mice compared with control mice as described previously (Fig. 1B, C and D) (Kitajima et al.2015). As a decrease in uterine size and increase in body weight are typical phenotypes of an estrogendeficient state (Jansson et al. 2006), our ovariectomy-induced estrogen-deficient model was established successfully.

\section{Estrogen insufficiency results in muscle atrophy and decreased muscle strength}

We next examined the effects of estrogen depletion on muscle functions. Although the muscle mass of the TA was unchanged, except at 8 weeks after ovariectomy (Fig. 2A), a grip test analysis showed a decrease in the muscle force generation of OVX mice at 24 weeks (Fig. 2B). To assess whether muscle fiber sizes were affected by estrogen insufficiency, we performed a histological

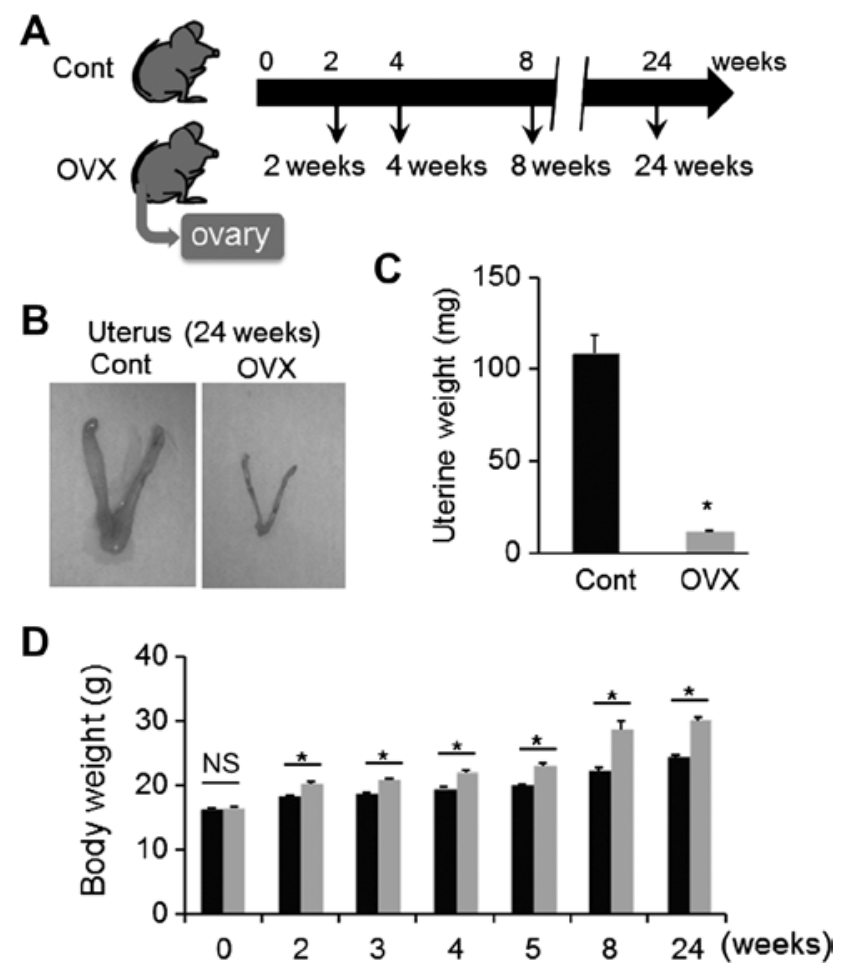

Figure 1

Estrogen-insufficient mouse model. (A) Six-week-old female C57BL/6 mice were ovariectomized (OVX) and sacrificed at 2, 4, 8, and 24 weeks after ovariectomy. Sham-operated mice were used as controls.

(B) Representative images of the uterus at 24 weeks after ovariectomy (quantified in (C)) (control, $n=8$; OVX, $n=6$ mice). Uterus weight was significantly decreased in OVX mice compared with control mice. (D) Body weight was increased gradually in OVX mice compared with control mice (control, $n=6-12$; OVX, $n=4-11$ mice). Data represent the mean \pm S.E.M. An asterisk denotes a significant difference from the control $\left({ }^{*} P<0.05\right)$. Scale bar: $100 \mu \mathrm{m}$. NS, not significant.
(C) 2016 Society for Endocrinology Printed in Great Britain 
analysis. Immunohistochemistry revealed that OVX mice exhibited a significant decrease in the cross-sectional area (CSA) of the TA muscle after 8 and 24 weeks compared with control mice (Fig. 2C, D and E). Despite the decrease in the CSA, muscle mass was increased at 8 weeks after ovariectomy. Thus, the amount of interstitial connective tissue may be altered in OVX muscle. Importantly, the ovariectomy-induced decline in CSA was completely rescued by treatment with $17 \beta$-estradiol at 8 weeks

A

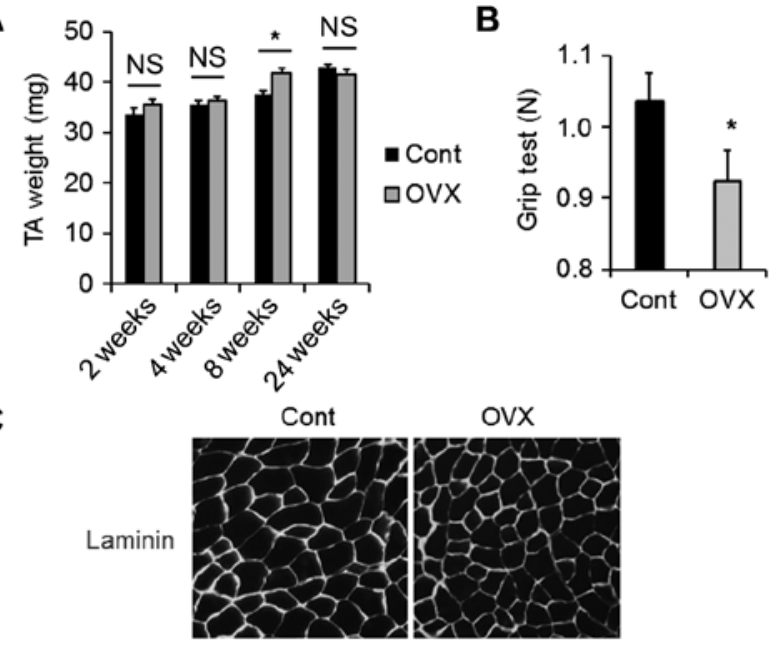

D

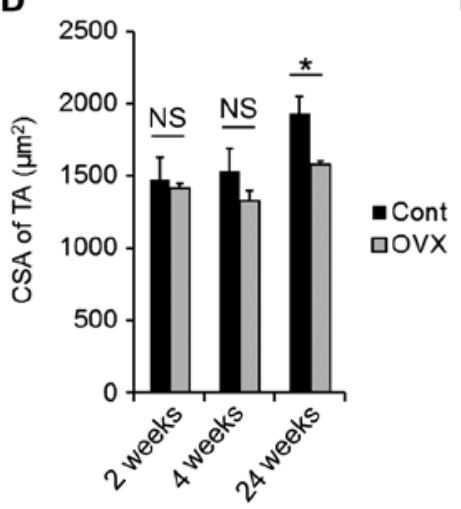

E

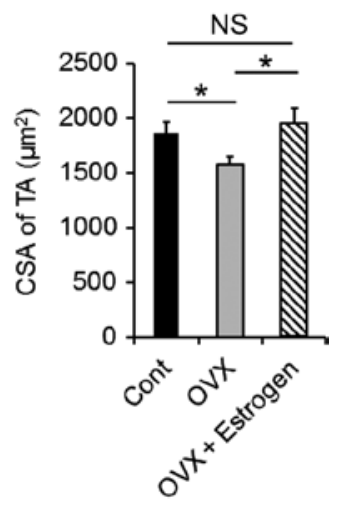

Figure 2

Ovariectomized (OVX) mice exhibit muscle atrophy and decreased muscle strength. (A) Muscle weight of the tibialis anterior (TA) muscle was measured at 2, 4, 8, and 24 weeks after OVX (control, $n=3-12$; OVX, $n=3-12$ mice). (B) Muscle force generation of forelimb muscle was measured by a grip strength meter at 24 weeks after OVX (control, $n=8$; OVX, $n=6$ mice). (C, D and E). Representative images of

immunohistochemistry for laminin at 24 weeks after ovariectomy (C) and the myofiber cross-sectional area (CSA) of TA muscle (D) (control, $n=3-4$; OVX, $n=3-4)$. Ovariectomy-induced decline of CSA was rescued by treatment with $17 \beta$-estradiol at 8 weeks after ovariectomy $(\mathrm{E})$ (control, $n=9$; OVX, $\mathrm{n}=9$; OVX + estrogen, $n=6$ mice) (at least 2000 myofibers per mouse were counted). Data represent the mean \pm S.E.M. An asterisk denotes a significant difference from the control $(* P<0.05)$. Scale bar: $100 \mu \mathrm{m}$. NS, not significant.
(Fig. 2E). These results indicate that estrogen insufficiency leads to muscle atrophy and decreased muscle strength of female mice.

\section{Reduced estrogens shift fiber types toward a faster distribution}

Considering the downregulation of muscle force generation in OVX mice, we next evaluated the fiber-type composition of muscle at 8 weeks. Immunohistochemistry showed an increase in the proportion of type IIb fibers in TA muscle under estrogen-deficient conditions (Fig. 3A and B). By contrast, the proportion of type IIa fibers was decreased in OVX mice. Importantly, we found that these fiber-type shifts were almost completely recovered by treatment with $17 \beta$-estradiol. We also showed a significant decrease in the CSA of all fast-type fibers (IIa, IIx, and IIb) at 24 weeks after ovariectomy (Fig. 3C). Thus, these findings suggest that estrogens prevent muscle atrophy, a shift toward faster MyHC isoform composition in female mice.

\section{Muscle stem cell functions are impaired in an estrogen-deficient state}

Satellite cells play crucial roles in providing myonuclei for postnatal muscle growth as well as muscle repair/ regeneration and hypertrophy in adults. Because accumulating evidence suggests that estrogens favor muscle regeneration after injury (Brown et al. 2009, Velders et al. 2012), we next determined the effects of prolonged estrogen insufficiency on satellite cell numbers and functions at 24 weeks after ovariectomy.

Satellite cells are mitotically quiescent in healthy adult muscle, but are activated in response to stimulation, such as muscle damage, to differentiate into myoblasts and proliferate extensively (Ono 2014). Quiescent satellite cells express the transcription factor Pax7 in adult muscle. We isolated murine satellite cells retained in their niche on myofibers isolated from the EDL muscle, and immunostained them for Pax7 as described previously (Masuda et al. 2015). Immunostaining showed no changes in the number of Pax $7^{+ \text {ve }}$ cells per fiber in OVX mice (Fig. 4A and B). Interestingly, we found an increase in the number of myonuclei per fiber in the estrogen-deficient state (Fig. 4A and C). This phenomenon was not observed in any other types of muscle weakness such as agerelated sarcopenia and disuse-induced muscle atrophy. The pathophysiological significance of this increase in

Published by Bioscientifica Ltd 
A

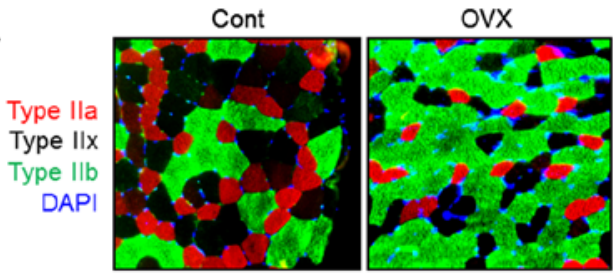

\section{B}

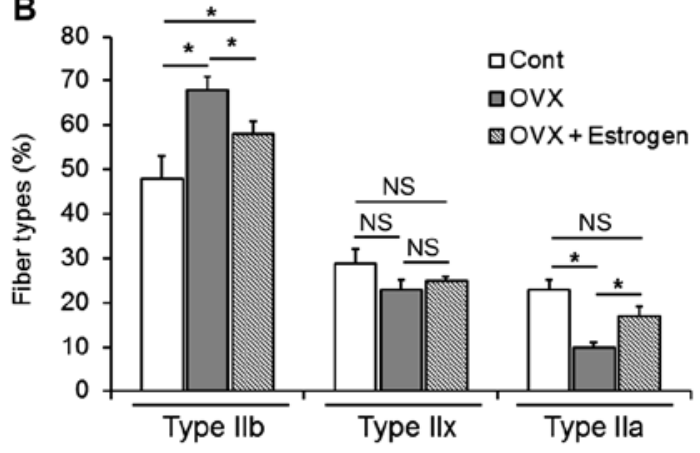

C

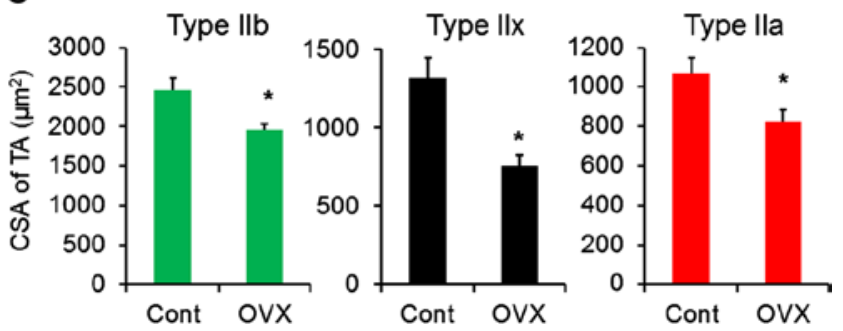

A
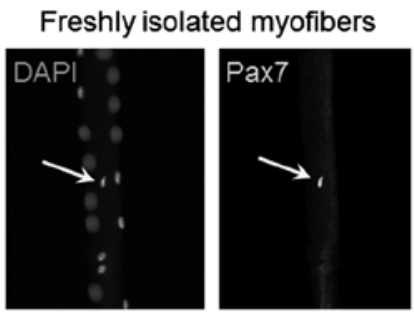

B

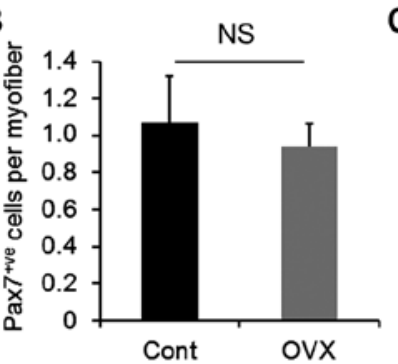

C

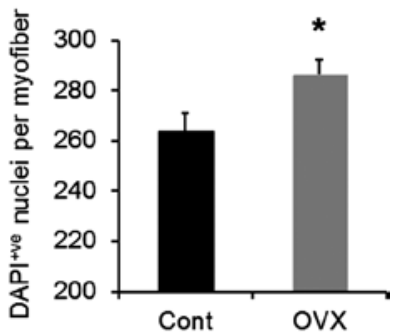

D

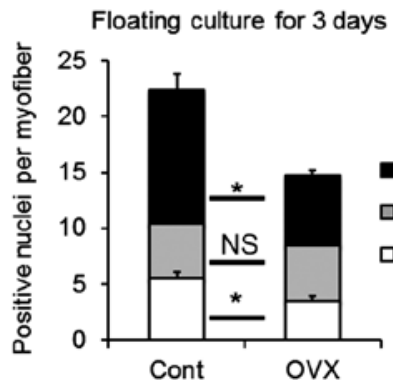

-Pax7-MyoD+ $\square \mathrm{Pax} 7+\mathrm{MyOD}+$ $\square P a x 7+M y o D-$

Figure 3

Estrogen insufficiency shifts fiber types toward faster MyHC isoforms. (A) Immunohistochemistry to visualize fiber types in TA muscle at 8 weeks after ovariectomy (quantified in $\mathrm{B}$, control, $n=6$; OVX, $n=5$; OVX+estrogen, $n=7$ ) (red, type Ila; black, type IIx; green, type IIb; blue, nucleus). (C) The CSA of individual fiber types in TA muscle was measured at 24 weeks after ovariectomy (control, $n=5$; OVX, $n=6-7$ ). The CSA of all three fast-type fibers (IIa, IIx, and IIb) was decreased in OVX mice. Data represent the mean \pm S.E.M. An asterisk denotes a significant difference from the control $\left({ }^{*} P<0.05\right)$. NS, not significant.

myonuclei in terms of muscle function was unclear. We speculate that increased myonuclear domain may play an important role in maintenance of muscle function in the estrogen insufficient state.

Satellite cell functions can be modeled in vitro (Moyle \& Zammit 2014). Upon stimulation by mitogenrich medium, quiescent satellite cells are activated, upregulate the myogenic regulatory factor MyoD, and then proliferate. Following expansion, satellite cells either downregulate Pax7, maintain MyoD, and undergo myogenic differentiation or downregulate MyoD, maintain Pax7, and return to a quiescent-like state, which models self-renewal (Zammit et al. 2004). Using this model, we examined whether satellite cell functions were influenced

\section{Figure 4}

Intrinsic satellite cell functions are impaired by estrogen insufficiency. $(A, B, C$, and $D)$ Satellite cells retained in their niche on myofibers were freshly isolated from the extensor digitorum longus (EDL) muscle at 24 weeks after OVX. (A) Representative images of immunostaining for Pax7. The numbers of Pax $7^{+ \text {ve }}$ satellite cells and DAPI+ve myonuclei per myofiber were quantified in (B) and (C), respectively (control, $n=4$; OVX, $n=6$ ).

(D) Satellite cells associated with myofibers were cultured in mitogen-rich medium for $72 \mathrm{~h}$, then co-immunostained for Pax7 and MyoD. Pax7+ve and/or MyoD+ve nuclei per myofiber were quantified. Pax7-veMyoD+ve differentiating and $\mathrm{Pax} 7^{+\mathrm{v}} \mathrm{MyoD}^{-\mathrm{ve}}$ self-renewing cell populations were decreased in OVX mice (control, $n=4$; OVX, $n=6$ ). Data represent the mean \pm S.E.M. An asterisk denotes a significant difference from the control $\left({ }^{*} P<0.05\right)$. NS, not significant.

by estrogen insufficiency. Satellite cells associated with myofibers were isolated from OVX mice and cultured in mitogen-rich medium for $72 \mathrm{~h}$. In our culture condition, culture media supplemented with chick embryo extract contain estrogens (Woods \& Brazzill 1981), and thus similarly influence the function of satellite cells from both control and OVX mice. Co-immunostaining for Pax7 and MyoD revealed a significant reduction in the total number of satellite cells from OVX mice. In particular, the proportions of differentiating Pax7-veMyoD+ve and self-renewing Pax7+veMyoD-ve satellite cells were significantly affected without a change in the proportion 
A

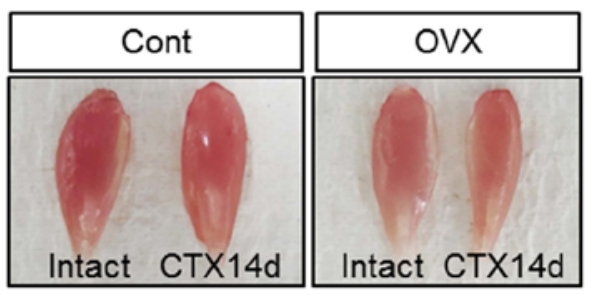

C
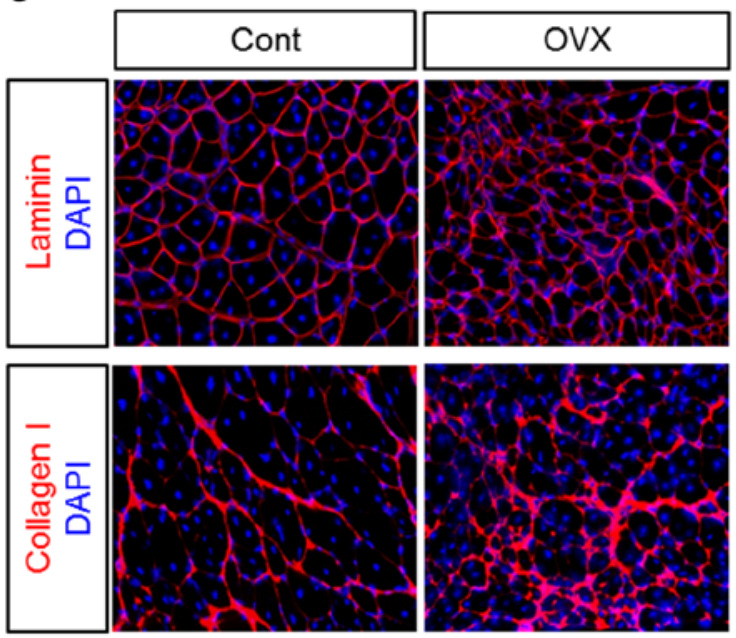

$\overline{100 \mu \mathrm{m}}$
B

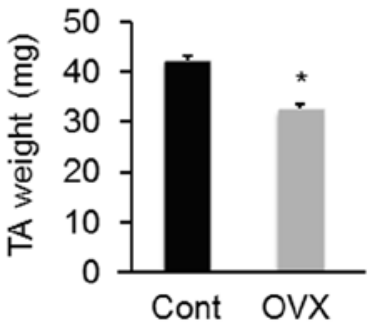

D

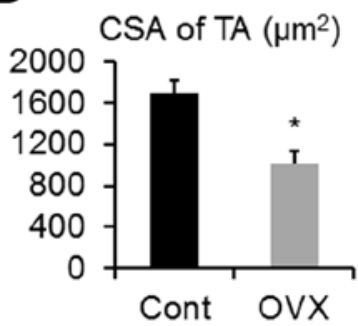

E

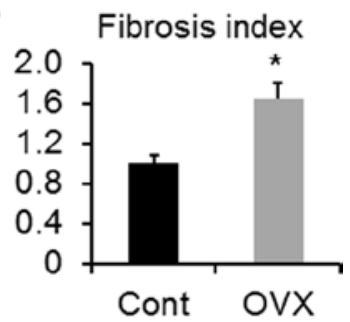

Figure $\mathbf{5}$

Estrogens are required for muscle regeneration. To induce muscle injury, cardiotoxin (CTX) was injected intramuscularly into TA muscle at 24 weeks after ovariectomy. Muscle regeneration was assessed by morphology, muscle weight, and immunohistochemistry of regenerating muscle at 14 days following CTX injection (CTX14d).

(A) Representative images of regenerating muscle. (B) Muscle weight was reduced in OVX mice at CTX14d (control, $n=8$; OVX, $n=6$ ). (C) Regenerating muscle cryosections were immunostained for laminin to analyze the CSA (D) or for collagen type I to assess fibrosis (E) (control, $n=5-6$; OVX, $n=6$ ). The estrogeninsufficiency state led to a marked decrease in muscle mass of OVX mice compared with control mice at CTX14d. Incomplete regeneration with fibrosis was observed in the muscle of OVX mice. Data represent the mean \pm S.E.M. An asterisk denotes a significant difference from the control $\left({ }^{*} P<0.05\right)$. Scale bar: $100 \mu \mathrm{m}$. of activated/proliferating Pax7+veMyoD+ve cells (Fig. 4D). Thus, these data imply that estrogens play an important role in maintaining the intrinsic function of satellite cells.

\section{Estrogens are indispensable for muscle regeneration}

As we found that satellite cell functions were impaired in OVX mice, we next evaluated the effect of estrogen insufficiency on muscle regeneration in vivo. CTX was used to induce muscle injury in the TA muscle of mice. Muscle regeneration was assessed by immunostaining of muscle sections at 14 days after injection of CTX (CTX14d) (Fig. 5A). An estrogen-deficient state led to a significant decrease in muscle weight compared with control mice at CTX14d (Fig. 5A and B). Cryosections of regenerating muscles were immunostained for laminin to analyze the CSA of regenerating myofibers or collagen type I to assess fibrosis. The CSA of centrally nucleated (regenerating) myofibers was markedly reduced in OVX mice compared with control mice (Fig. 5C, quantified in 5D). Immunohistochemistry also revealed incomplete regeneration with marked fibrosis in the muscle of OVX mice (Fig. 5C, quantified in 5E). Taken together, these data suggest that estrogens are required for muscle regeneration in vivo by affecting efficient population expansion of satellite cells during regeneration.

\section{Discussion}

Skeletal muscle is a highly plastic tissue that readily adapts its contractile and metabolic properties in response to muscle activities. How the plasticity of skeletal muscle is controlled is pivotal to understanding the underlying mechanisms of muscle-wasting diseases (Egerman \& Glass 2014, Cohen et al. 2015). In this study, we examined the effects of ovariectomy-induced estrogen reduction on muscle and satellite cells. OVX rats fail to efficiently recover muscle mass after unloading followed by reloading compared with OVX rats treated with estrogens (McClung et al. 2006, Sitnick et al. 2006), suggesting that estrogens are important for muscle regrowth. By contrast, estrogen receptor alpha (Er $(E s r 1))$-, but not $\operatorname{Er} \beta$ (Esr2)- knockout female mice display a significant increase in absolute muscle weight (Brown et al. 2009). However, such ER $\alpha$ inactivation results in a decrease in the muscle peak tetanic tension per CSA (Brown et al. 2009). In agreement with these observations, we found that OVX mice exhibited both an increase in the absolute muscle mass of the TA muscle and a reduction in muscle force generation. We also showed that the ovariectomy-induced estrogendeficient state resulted in a significant decline in the fiber CSA of the TA muscle. However, no effect on the fiber

Published by Bioscientifica Ltd. 
CSA of the slow-twitch soleus muscle has been observed between OVX and sham-operated female rats (McClung et al. 2006). In addition, knockdown of Era in hindlimb muscles increases the weight of soleus muscle in females (Ogawa et al. 2015). These discrepancies indicate that estrogens influence fiber atrophy specifically in fast-type muscle, such as TA muscle, but not in slow-type muscle.

Menopause is defined as permanent termination of menstruation. A marked decline in estrogen levels is observed in the late stage of menopause transition (Messier et al. 2011). The decrease in estrogen levels leads to a reduction in bone mass density and an increase in the white fat mass (Mauvais-Jarvis 2011). A crosssectional study reported a decline in muscle mass of $0.6 \%$ per year following menopause (Rolland et al. 2007). In this study, our ovariectomy model using young mice showed an estrogen-insufficient state that resulted in a significantly atrophic uterus and increased body weight, which partially mimicked a postmenopausal state. In general, fast-to-slow fiber-type conversion is found in muscle with aging (Schiaffino \& Reggiani 2011). We thus expected to observe phenotypes similar to aged muscle in our ovariectomy model. Interestingly, however, estrogen insufficiency resulted in a shift toward a faster fiber-type profile in the TA. This finding is not consistent with the previous rat study, in which ovariectomy induced a shift from fast-to-slow MyHC isoforms in both soleus and EDL muscle (Kadi et al. 2002). This discrepancy may be explained by the different experimental conditions. Kadi and coworkers measured MyHC contents in homogenized whole muscle by gel electrophoresis, whereas we evaluated the proportions of fiber types by immunohistochemistry. In fact, our quantitative RT-PCR analysis did not show any differences in the levels of gene expression for MyHC isoforms in homogenized whole muscle between OVX and control mice (data not shown).

Although we did not assess the molecular mechanism how prolonged estrogen insufficiency induces muscle atrophy and a shift to a faster fiber-type distribution, similar phenomena have been described in diseases involved in inflammatory responses, such as cancer cachexia, chronic obstructive pulmonary disease (COPD), and chronic heart failure (CHF) (Holecek 2012, Roberts et al. 2013). For example, a shift toward a faster MyHC isoform composition is observed in the muscles of patients with COPD and CHF (Ciciliot et al. 2013). Insufficient oxygen delivery to peripheral tissues can be seen in COPD and CHF, resulting in hypoxia in skeletal muscle (Gosker et al. 2000). Hypoxic conditions are well known to induce the expression of hypoxia-inducible factor $1 \alpha(\mathrm{HIF} 1 \alpha)$ in tissues including muscle (Stroka et al. 2001). Overexpression of HIF $1 \alpha$ leads to slow-to-fast fiber conversion (Lunde et al. 2011), thus seemingly linking to the phenotypes of muscles in COPD and CHF. A recent study has reported that HIF $1 \alpha$ is a crucial factor in the activation of osteoclasts that promote bone resorption after menopausal estrogen insufficiency. Indeed, estrogens prevent HIF $1 \alpha$ activation, leading to the suppression of osteoclast activity (Miyauchi et al. 2013). Nuclear factor- $\mathrm{kB}$ (NF-kB), a transcription factor of inflammatory genes, induces muscle atrophy, along with the loss of slow-twitch fibers (Li et al. 2008, Wang \& Pessin 2013). Estrogens suppress inflammation responses by inhibiting the activation of NF-кB (Stice \& Knowlton 2008). Considering these findings, the effect of estrogen insufficiency on the properties of muscle might be involved in the activation of HIF1 $\alpha$ and NF- $\mathrm{KB}$ in the muscle of our ovariectomy model.

Accumulating evidence demonstrates that estrogens favor muscle regeneration after injury (Diel 2014). Treatment with estrogens attenuates exercise-induced muscle injury (Tiidus et al. 2001), which may be associated with upregulation of the number of satellite cells and promotion of satellite cell proliferation (Tiidus et al. 2005, Enns \& Tiidus 2008). This enhanced satellite cell activity induced by estrogen supplementation appears to be mediated through ER $\alpha$ and/or ER $\beta$ (Velders et al. 2012). As the above studies evaluated the function of satellite cells by histological analysis in vivo, whether extrinsic factors in the regenerating niche influence satellite cell activity or the intrinsic regenerative potential of satellite cells is affected by estrogen insufficiency remains unclear. In this study, our single myofiber method revealed no alteration in the number of satellite cells per myofiber, while population expansion, differentiation, and self-renewal were significantly impaired in satellite cells isolated from OVX mice upon culture in vitro. Importantly, we confirmed that muscle regeneration is severely compromised in OVX mice. Taken together, our results demonstrated for the first time that the intrinsic function of satellite cells is diminished by prolonged estrogen insufficiency in females. However, it remains unclear how estrogens maintain the intrinsic function of satellite cells.

In conclusion, our results show that prolonged estrogen insufficiency induces not only muscle atrophy accompanied by slow-to fast-type conversion, but also intrinsic dysfunction of satellite cells in muscle. Indeed, estrogens are essential for comprehensively maintaining muscle function with its insufficiency affecting muscle strength and regeneration in females. These findings may be relevant to a higher risk of sarcopenia in older postmenopausal women (Sørensen

Published by Bioscientifica Ltd. 
et al. 2001). It is also important to note that, especially in endurance sports such as marathons, which mainly demand slow-twitch muscle rather than fast-twitch muscle, estrogen deficiency may therefore give rise to severe problems in the exercise performance of female athletes. Further studies are needed to understand the molecular mechanisms of how estrogen insufficiency influences muscle and satellite cell properties in female.

\section{Declaration of interest}

The authors declare that there is no conflict of interest that could be perceived as prejudicing the impartiality of the research reported.

\section{Funding}

This work was supported by Special Coordination Funds for Promoting Science and Technology from the Japan Science and Technology Agency (JST), a Grant-in-Aid for Challenging Exploratory Research (Research Project Number 25560338) and a Grant-in-Aid for Young Scientists (A) (Research Project Number 15H05368) from the Japan Society for the Promotion of Science (JSPS), and the Sasagawa Scientific Research Foundation.

\section{Author contribution statement}

$\mathrm{Y} K$ designed and performed the experiments, analyzed the data, and wrote the manuscript. $Y O$ designed and performed the experiments, analyzed and interpreted the data, assembled the input data, and wrote the main manuscript. All authors discussed the results and implications, and commented on the manuscript.

\section{Acknowledgments}

The authors thank Ms Yumiko Takemoto, Drs Shinya Masuda, Shizuka Ogawa, Yoshishige Urata, Shinji Goto, Tao-Sheng Li, and Hideaki Masuzaki for technical assistance and financial support.

\section{References}

Brown M, Ning J, Ferreira JA, Bogener JL \& Lubahn DB 2009 Estrogen receptor-alpha and -beta and aromatase knockout effects on lower limb muscle mass and contractile function in female mice. American Journal of Physiology Endocrinology and Metabolism 296 E854-E861. (doi:10.1152/ajpendo.90696.2008)

Ciciliot S, Rossi AC, Dyar KA, Blaauw B \& Schiaffino S 2013 Muscle type and fiber type specificity in muscle wasting. International Journal of Biochemistry and Cell Biology 45 2191-2199. (doi:10.1016/j. biocel.2013.05.016)

Cohen S, Nathan JA \& Goldberg AL 2015 Muscle wasting in disease: molecular mechanisms and promising therapies. Nature Reviews Drug Discovery 14 58-74. (doi:10.1038/nrd4467)

Collins CA \& Zammit PS 2009 Isolation and grafting of single muscle fibres. Methods in Molecular Biology 482 319-330. (doi:10.1007/978-159745-060-7)

Diel P 2014 The role of the estrogen receptor in skeletal muscle mass homeostasis and regeneration. Acta Physiologica 212 14-16. (doi:10.1111/apha.12341)
Duckham RL, Peirce N, Meyer C, Summers GD, Cameron N \& BrookeWavell K 2012 Risk factors for stress fracture in female endurance athletes: a cross-sectional study. BMJ Open 2 e001920. (doi:10.1136/ bmjopen-2012-001920)

Dumont NA, Bentzinger CF, Sincennes MC \& Rudnicki MA 2015 Satellite cells and skeletal muscle regeneration. Comprehensive Physiology 5 1027-1059. (doi:10.1002/cphy.c140068)

Egerman MA \& Glass DJ 2014 Signaling pathways controlling skeletal muscle mass. Critical Reviews in Biochemistry and Molecular Biology 49 59-68. (doi:10.3109/10409238.2013.857291)

Enns DL \& Tiidus PM 2008 Estrogen influences satellite cell activation and proliferation following downhill running in rats. Journal of Applied Physiology 104 347-353. (doi:10.1152/ japplphysiol.00128.2007)

Gosker HR, Wouters EF, van der Vusse GJ \& Schols AM 2000 Skeletal muscle dysfunction in chronic obstructive pulmonary disease and chronic heart failure: underlying mechanisms and therapy perspectives. American Journal of Clinical Nutrition 71 1033-1047.

Holecek M 2012 Muscle wasting in animal models of severe illness. International Journal of Experimental Pathology 93 157-171. (doi:10.1111/j.1365-2613.2012.00812.x)

Jansson JO, Moverare-Skrtic S, Berndtsson A, Wernstedt I, Carlsten H \& Ohlsson C 2006 Leukemia inhibitory factor reduces body fat mass in ovariectomized mice. European Journal of Endocrinology 154 349-354. (doi:10.1530/eje.1.02082)

Kadi F, Karlsson C, Larsson B, Eriksson J, Larval M, Billig H \& Jonsdottir IH 2002 The effects of physical activity and estrogen treatment on rat fast and slow skeletal muscles following ovariectomy. Journal of Muscle Research and Cell Motility 23 335-339. (doi:10.1023/A:1022071114344)

Kitajima Y, Doi H, Ono Y, Urata Y, Goto S, Kitajima M, Miura K, Li TS \& Masuzaki H 2015 Estrogen deficiency heterogeneously affects tissue specific stem cells in mice. Scientific Reports 5 12861. (doi:10.1038/ srep12861)

Li H, Malhotra S \& Kumar A 2008 Nuclear factor-kappa B signaling in skeletal muscle atrophy. Journal of Molecular Medicine 86 1113-1126.

Lunde IG, Anton SL, Bruusgaard JC, Rana ZA, Ellefsen S \& Gundersen K 2011 Hypoxia inducible factor 1 links fast-patterned muscle activity and fast muscle phenotype in rats. Journal of Physiology 5891443 1454. (doi:10.1113/jphysiol.2010.202762)

Masuda S, Hisamatsu T, Seko D, Urata Y, Goto S, Li TS \& Ono Y 2015 Time- and dose-dependent effects of total-body ionizing radiation on muscle stem cells. Physiological Reports 3 e12377.

Matzkin E, Curry EJ \& Whitlock K 2015 Female athlete triad: past, present, and future. Journal of the American Academy of Orthopaedic Surgeons 23 424-432. (doi:10.5435/JAAOS-D-14-00168)

Mauvais-Jarvis F 2011 Estrogen and androgen receptors: regulators of fuel homeostasis and emerging targets for diabetes and obesity. Trends in Endocrinology and Metabolism 22 24-33. (doi:10.1016/j.tem.2010.10.002)

McClung JM, Davis JM, Wilson MA, Goldsmith EC \& Carson JA 2006 Estrogen status and skeletal muscle recovery from disuse atrophy. Journal of Applied Physiology 100 2012-2023. (doi:10.1152/ japplphysiol.01583.2005)

Messier V, Rabasa-Lhoret R, Barbat-Artigas S, Elisha B, Karelis AD \& Aubertin-Leheudre M 2011 Menopause and sarcopenia: a potential role for sex hormones. Maturitas 68 331-336. (doi:10.1016/j. maturitas.2011.01.014)

Miyauchi Y, Sato Y, Kobayashi T, Yoshida S, Mori T, Kanagawa H, Katsuyama E, Fujie A, Hao W, Miyamoto K, et al. 2013 HIF1alpha is required for osteoclast activation by estrogen deficiency in postmenopausal osteoporosis. PNAS 110 16568-16573. (doi:10.1073/ pnas.1308755110)

Moyle LA \& Zammit PS 2014 Isolation, culture and immunostaining of skeletal muscle fibres to study myogenic progression in satellite cells Methods in Molecular Biology 1210 63-78. (doi:10.1007/978-1-49391435-7)

Published by Bioscientifica Ltd. 
Ogawa M, Kitakaze T, Harada N \& Yamaji R 2015 Female-specific regulation of skeletal muscle mass by USP19 in young mice. Journal of Endocrinology 225 135-145. (doi:10.1530/JOE-15-0128)

Ono Y 2014 Satellite cell heterogeneity and hierarchy in skeletal muscle. Journal of Physical Fitness and Sports Medicine 3 229-234. (doi:10.7600/ jpfsm.3.229)

Ono Y, Calhabeu F, Morgan JE, Katagiri T, Amthor H \& Zammit PS 2011 BMP signalling permits population expansion by preventing premature myogenic differentiation in muscle satellite cells. Cell Death and Differentiation 18 222-234. (doi:10.1038/cdd.2010.95)

Pollanen E, Sipila S, Alen M, Ronkainen PH, Ankarberg-Lindgren C, Puolakka J, Suominen H, Hamalainen E, Turpeinen U, Konttinen YT, et al. 2011 Differential influence of peripheral and systemic sex steroids on skeletal muscle quality in pre- and postmenopausal women. Aging Cell 10 650-660. (doi:10.1111/j.14749726.2011.00701.x)

Rauh MJ, Nichols JF \& Barrack MT 2010 Relationships among injury and disordered eating, menstrual dysfunction, and low bone mineral density in high school athletes: a prospective study. Journal of Athletic Training 45 243-252. (doi:10.4085/1062-6050-45.3.243)

Roberts BM, Frye GS, Ahn B, Ferreira LF \& Judge AR 2013 Cancer cachexia decreases specific force and accelerates fatigue in limb muscle. Biochemical and Biophysical Research Communications 435 488-492. (doi:10.1016/j.bbrc.2013.05.018)

Rolland YM, Perry HM III, Patrick P, Banks WA \& Morley JE 2007 Loss of appendicular muscle mass and loss of muscle strength in young postmenopausal women. Journal of Gerontology A: Biological Sciences and Medical Sciences 62 330-335. (doi:10.1093/gerona/62.3.330)

Schiaffino S \& Reggiani C 2011 Fiber types in mammalian skeletal muscles. Physiological Reviews 91 1447-1531. (doi:10.1152/ physrev.00031.2010)

Sitnick M, Foley AM, Brown M \& Spangenburg EE 2006 Ovariectomy prevents the recovery of atrophied gastrocnemius skeletal muscle mass. Journal of Applied Physiology 100 286-293. (doi:10.1152/ japplphysiol.00869.2005)
Sørensen MB, Rosenfalck AM, Højgaard L, Ottesen B 2001 Obesity and sarcopenia after menopause are reversed by sex hormone replacement therapy. Obesity Research 9 622-626. (doi:10.1038/oby.2001.81)

Stice JP \& Knowlton AA 2008 Estrogen, NFkappaB, and the heat shock response. Molecular Medicine 14 517-527. (doi:10.2119/2008-00026.stice)

Stroka DM, Burkhardt T, Desbaillets I, Wenger RH, Neil DA, Bauer C, Gassmann M \& Candinas D 2001 HIF-1 is expressed in normoxic tissue and displays an organ-specific regulation under systemic hypoxia. FASEB Journal 15 2445-2453. (doi:10.1096/fj.01-0125com)

Sugiura T, Ito N, Goto K, Naito H, Yoshioka T \& Powers SK 2006 Estrogen administration attenuates immobilization-induced skeletal muscle atrophy in male rats. Journal of Physiological Sciences 56 393-399. (doi:10.2170/physiolsci.RP006906)

Tiidus PM, Deller M \& Liu XL 2005 Oestrogen influence on myogenic satellite cells following downhill running in male rats: a preliminary study. Acta Physiologica Scandinavica 184 67-72. (doi:10.1111/j.1365201x.2005.01427.x)

Tiidus PM, Holden D, Bombardier E, Zajchowski S, Enns D \& Belcastro A 2001 Estrogen effect on post-exercise skeletal muscle neutrophil infiltration and calpain activity. Canadian Journal of Physiology Pharmacology 79 400-406. (doi:10.1139/y01-011)

Velders M, Schleipen B, Fritzemeier KH, Zierau O \& Diel P 2012 Selective estrogen receptor-beta activation stimulates skeletal muscle growth and regeneration. FASEB Journal 26 1909-1920. (doi:10.1096/fj.11194779)

Wang Y \& Pessin JE 2013 Mechanisms for fiber-type specificity of skeletal muscle atrophy. Current Opinion in Clinical Nutrition and Metabolic Care 16 243-250. (doi:10.1097/MCO.0b013e328360272d)

Woods JE \& Brazzill DM 1981 Plasma 17 beta-estradiol levels in the chick embryo. General and Comparative Endocrinology 44 37-43. (doi:10.1016/0016-6480(81)90353-1)

Zammit PS, Golding JP, Nagata Y, Hudon V, Partridge TA \& Beauchamp JR 2004 Muscle satellite cells adopt divergent fates: a mechanism for self-renewal? Journal of Cell Biology 166 347-357. (doi:10.1083/ jcb.200312007)

Received in final form 30 March 2016

Accepted 5 April 2016

Accepted Preprint published online 5 April 2016 http://joe.endocrinology-journals.org DOI: $10.1530 / J O E-15-0476$
(C) 2016 Society for Endocrinology Printed in Great Britain
Published by Bioscientifica Ltd. 